$\mathrm{KN}-7$

\section{Biomolecular structure determination by electron diffraction of 3D microcrystals}

Brent Nannenga ${ }^{1}$

1. Chemical Engineering, Arizona State University, Tempe, United States of America

email: Brent.Nannenga@asu.edu

A common barrier to high-resolution biomolecular structure determination is the growth of large well-ordered crystals. Electron diffraction is capable of producing high-quality diffraction data from crystals that are orders of magnitude smaller than those needed for conventional X-ray crystallographic experiments, and 3D electron diffraction methods have recently begun to yield high-resolution structures from extremely small microcrystals. In this presentation, the cryo-electron microscopy technique of microelectron diffraction, or MicroED, will be described in detail along with representative structures determined by the method. Additionally, current work in our lab, which is focused on improving and extending this technique to very difficult samples, such as membrane proteins, will be presented.

Keywords: Electron diffraction, protein crystallography
KN-8

\section{Exotic topochemical alteration of the cationic sublattices in oxides: the complex crystallography behind}

\author{
Olivier Mentré ${ }^{1}$ \\ 1. UCCS, UMR CNRS 8181, Villeneuve D'ascq, France \\ email: olivier.mentre@ensc-lille.fr
}

The possibility to modify inorganic oxides at moderate temperature, under kinetic rather than thermodynamic control leads to metastable structural rearrangements with novel electronic partitions and properties. Here we present a review of recent exotic reversible topochemical reactions studied in the lab. In most cases, the observed modidication concerns the anionic sub-array. However we have evidenced a number of structural lattices in which the cationic lattices itself may be drastically re-arranged via concomitant or not alteration of the anionic sublattice. For instance, the controlled oxidation of the 2D-ising ferromagnetic $\mathrm{BaFe}_{2}{ }_{2}\left(\mathrm{PO}_{4}\right)_{2}$ into Fe-depleted $\mathrm{BaFe}^{2 / 3+}{ }_{2-\mathrm{x}}\left(\mathrm{PO}_{4}\right)_{2}$ $(\mathrm{x}<0.66)$ leads to a series of intermediate phases with full vacancy/Fe ordering and to nanometric $\mathrm{Fe}_{2} \mathrm{O}_{3}[1]$. Here, antiferromagneticexchanges are recovered along with the $\mathrm{Fe}^{2+}$ to $\mathrm{Fe}^{3+}$ oxidation. Other pertinent examples of metal exsolution will be discussed. On the opposite, playing redox chemistry in 2D-oxides such as the multiferroic hexagonal $\mathrm{YMn}^{3+} \mathrm{O}_{3}, \mathrm{YbFe}^{2.5+}{ }_{2} \mathrm{O}_{4}$ and $\mathrm{Yb}_{2} \mathrm{Fe}^{2.66+}{ }_{3} \mathrm{O}_{7}$, the metal content is maintained but drastically restacked during reduction/oxidation into $\mathrm{YMn}^{2+} \mathrm{O}_{2.5} \mathrm{YbFe}^{3+}{ }_{2} \mathrm{O}_{4.5}$ and $\mathrm{Yb}_{2} \mathrm{Fe}^{3+}{ }_{3} \mathrm{O}_{7.5}[2,3]$. Generally, all transformed compounds require complex crystal-chemistry features with occurence of supercells, modulated structures, and/or disordred intregrowths.

The possibility to tune in a controlled way various pristine frameworks opens a wide field of investigation for tailor-made crystallographic architectures.This fascinating crystal chemistry upon oxidation and reduction will be described and rationalized for a global vision of these giant anion/cation-labile systems.

References:

[1] I. Blazquez Alcover. et al. Crystal Growth and Design, (2015).

15,4237 ,

[2] S. Nicoud et al.. JACS, (2017).139, 17031,

[3] H. Kabbour et al.. Inorg. chem. (2017), 56, 8547.

Keywords: metal exsolution, topochemical transformation, metal depleted lattices 